\title{
Decision support systems in preliminary design
}

\author{
Patrick Sebastian • Yann Ledoux
}

Published online: 18 September 2009

(C) Springer-Verlag 2009

\section{Introduction}

Many applications of the engineering design are facing with the development of decision support systems. This challenging issue is especially prominent in the preliminary phases of design. Mechanical engineering, Energy engineering, Process engineering, Material engineering and Design or Manufacturing are all concerned with the development of decision support systems. Preliminary Design is interested in the search for innovative concepts as well as in the optimization of systems, parts or materials. This global optimization process is concerned with the optimization of shapes, geometries, structures, dimensions, material combinations, etc. Recent progresses have been performed in the development of tools supporting decision making in the search for innovative concepts or in the development of numerical methods suitable for the optimization of complex mechanical systems in the early phases of their design.

This special issue focuses on advances in design heuristics, numerical methods inspired by nature (genetic algorithms, simulated annealing, particle swarms, etc.), Monte Carlo or stochastic methods and Constraint Programming for design applications. Some approaches are "qualitative" in the sense that they provide reasoning mechanisms to optimize concepts; other approaches are "quantitative" being more numerically oriented to carry out the translation of concepts into quantified design variables or decision variables.

Preliminary design phases have been defined by Pahl and Beitz in the early 1980's as the phases of the design process

\section{P. Sebastian $(\varangle) \cdot$ Y. Ledoux}

Université de Bordeaux, TREFLE Laboratory,

Esplanade des Arts et Métiers, 33400 Talence, France

e-mail: patrick.sebastian@Bordeaux.Ensam.fr

Y. Ledoux

e-mail: yann.ledoux@u-bordeaux1.fr which aim at defining the design requirements, the concepts (conceptual design) and the main characteristics of the product (embodiment design). The embodiment design phase is challenging in the context of industrial design departments. Even if the knowledge is uncertain at the beginning of this design phase, $70 \%$ of life-cycle costs are engaged by decisions at the end of preliminary design. This phase (see Fig. 1) leads to the expression of a design configuration (product architecture) where main dimensions and components are chosen.

Most of the existing computer aided tools are not suitable for supporting early design phases because they are based on models requiring the complete geometrical definition of the product (local optimization). Designers do not have the tools to assist them in making decisions with regard to the choice of concepts whose performances they have to assess.

Therefore, many a priori choices are performed according to the expertise of the designer or of the company; these choices hide a great part of the potential solution space. Finally, the dimensioning process of the system is performed for the most critical life-cycle stage, the prevailing situation. Using a trial and error mode, the designer converges towards a solution which is not necessarily the most powerful, or, which doesn't correspond to the best trade off between the various functioning phases and the expected product performances.

Embodiment design problem can be naturally expressed as mixed Constraint Satisfaction Problems (CSP); which cannot be solved using classical mechanical simulation tools. The design problem is described (see Fig. 2) by a set of design variables (DV), criteria and constraints:

The constraints can be written as:

- Relations (equalities, inequalities, logical rules); 


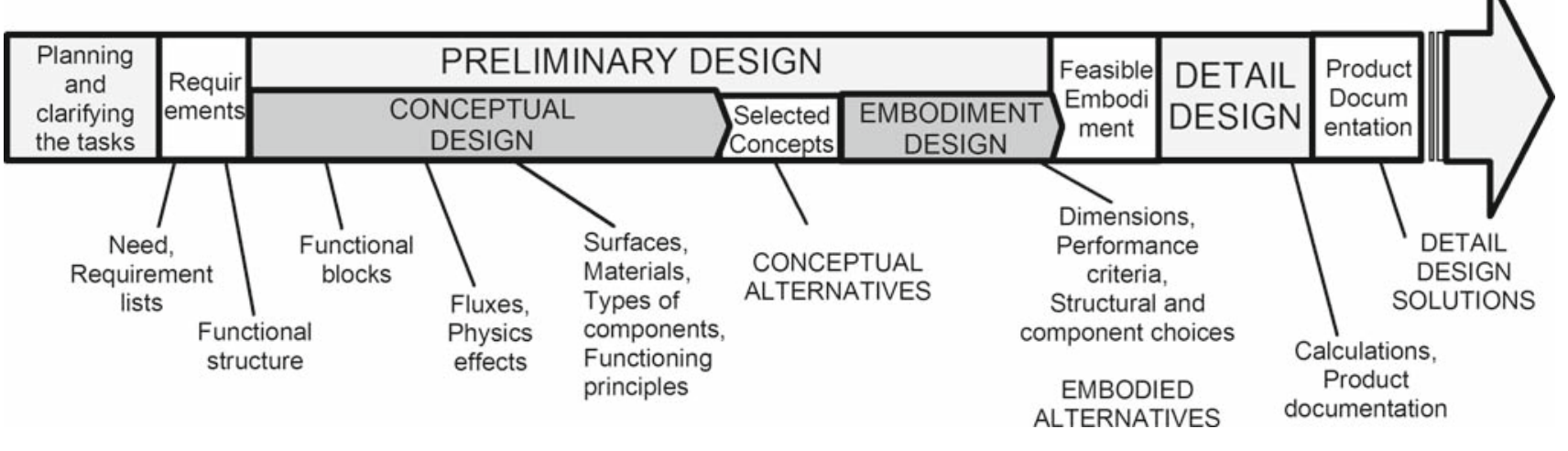

Fig. 1 Evolution of artifacts and product data of each design process phase

Fig. 2 From design problem models to the feasible embodiments

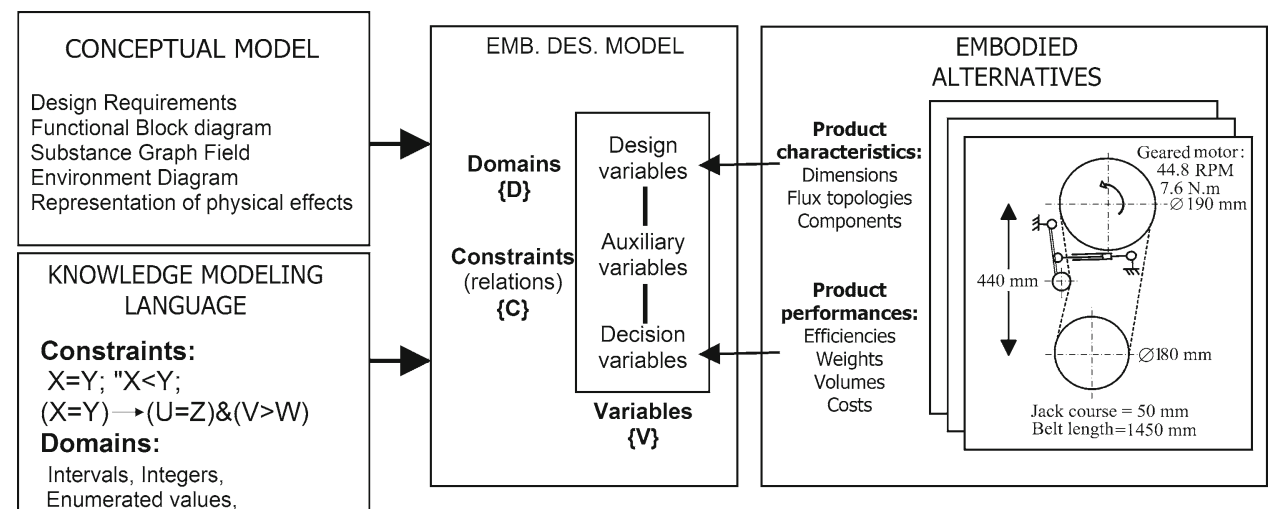

- Variable domains: continuous (dimensions, T, P, etc.), discrete (structure, topology), enumerated (materials), tabulated (standard components).

The constraints are related to:

- The requirements and the functional performance specifications,

- The technical skills and manufacturing rules,

- The physical behaviour models (conservation laws) in order to describe the functional fluxes but also the undesirable induced phenomena,

- The reliability to standard concepts or catalogues of industrial products,

- The life cycle stages (different functioning situations).

The main variable types in the model are:

- The Design Variables (DV): they allow differentiating the design configurations between them (dimension, standard component characteristics, materials, mass, number of elements, etc.). These different values for each design variable totally define particular product architecture.
- The Performance Variables (PV): they translate the properties, the state or the quality of a design configuration and allow to qualify the level of performance according to the customer or the designer point of views.

Digital processing tools based on different types of numerical approaches have recently been developed to cope with Constraint Satisfaction Problems relating to preliminary design. Most of these tools are concerned with interval analysis techniques, evolutionary programming techniques and MonteCarlo methods for design reliability and robustness.

\section{Search of feasible design solutions: tools and techniques}

Constraint Satisfaction Problem solving based on interval analysis techniques aim at jointly solving design requirements, physical phenomena and knowledge relating to the life cycle of the product. Preliminary design models are regarded as sets of variables (V), domains (D) and constraints (C). A domain is a set of numerical values linked to a variable which bounds the feasible values inside of a compact domain 
corresponding to interval of floating points or a list (sequential or not). A constraint is a relation which interrelate the variables and the domain of values of the variables. Relations of a Constraint Satisfaction Problem may involve algebra, analytical functions, differential equations, dynamic programming, and so on.

Mixed CSPs tackle with the numerical solving of problems which mix continuous and discrete domains of values. For instance, the design of wind turbine systems may require the calculation of dimensions (blades, mast, etc.), power coefficients, etc. These variables must be calculated on continuous domains (real numbers). On the other hand, the values of some variables may have to be selected among lists or calculated inside of sequential domains. These variables are related to discrete domain of values and concern, as an example, the number of blades of a wind turbine (integer numbers) or the type of control of the wind turbine (Pitch, Stall, etc.). Most CSP solvers are concerned with discrete problems; namely, these problems only take into account discrete variable domains. Recent progresses in the domain of CSPs concern the development of numerical methods suitable for continuous problems. These numerical methods are based on interval analysis techniques. These methods easily are extended to mix CSPs (continuous/discrete) and prove to remain efficient provided that most of the variables evolve on continuous domains.

The basic algorithm underlying these techniques is to initiate the calculation of every variable domain of the model from a wide compact and continuous domain (interval) and to gradually prune inconsistent values from these initial domains in order to decrease their size. This pruning process is stopped when:

- the interval domains are empty (no solution),

- the size of the interval domains are small enough and reach a predefined precision (solution),

- the pruning method is trapped and can't decrease the size of the interval domains.

In the third case, the interval domains are split into smaller interval domains to restart the process on several sub-problems. These sub-problems are regarded as branches (subproblems) of a tree (the Constraint Satisfaction Problem) and the numerical method is based on a branch and bound algorithm. The exploration process of the design problem is complete when the algorithm sequentially explores every branch of the problem and doesn't stop until it finds every solution of the problem.

This approach has several potential benefits regarding decision support in the preliminary phases of the design of mechanical systems. First, the models of design problems of mechanical systems are heterogeneous. They mix several types of constraints (equalities, inequalities, etc.) and sev- eral types of variables (continuous, sequential, lists, etc.). This heterogeneity derives from the nature of the constraints which are taken into consideration during the preliminary phases of the design process. Interval analysis based algorithms are much less sensitive to this heterogeneity than classical exploration algorithms since they are lowly sensitive to non linearity and to the disjunctions of the model. Additionally, the exploration process is complete whereas most of the exploration methods only investigate some parts of the design solution space. Interval analysis may very quickly prove the lack of solutions in some wide parts of the exploration space. This property brings out the interest and the significance of these numerical methods during the preliminary phases of the design of wind turbines. It is used to rapidly discriminate non-feasible configurations of systems regarding the design requirements of the project.

Most of the classical optimization methods are based on differential or variational calculus. Indeed, in most of the optimization problems the objective function appears to be continuous and derivable, then optimal values of the function may be calculated using iterative methods. These methods are limited to the treatment of optimization problems leading to continuous, derivable and well-defined objective functions. Since the mid-1950's, more difficult problems have been handled in the artificial intelligence filed. These optimization problems are solved using knowledge-based systems, fuzzy logic, inductive learning, neural networks and genetic algorithms.

Owing their efficiency and robustness in the global optimization of design problems involving non-linear and non-explicit variables, evolutionary techniques are used as optimization techniques. The use of evolutionary optimization techniques is relevant since the embodiment design of mechanical systems is a combinatorial problem and the number of possible configurations of system is very substantial.

Genetic algorithms, developed by Holland, are powerful tools for the global optimization of problems defined through non-continuous variables and functions. They are well adapted to the solution of non-linear problems that lead to objective function containing many local minima or maxima. Objective functions may be non derivable or ill-defined (as the result of a complex algorithm for instance), and no gradient information is required to solve them. More to the point, genetic algorithms appear to be robust and easy to implement. Classical optimization method, e.g. gradient, Newton or quasi-Newton methods are not suitable for the optimization of such hard-to-solve problems. Genetic algorithm implementation is based on the evolution of a population including several individuals towards a best individual by using selection and natural reproduction processes. These processes are based on the Darwinian concept of natural selection. Individual corresponds to candidate solutions of the embodiment design problem. 


\section{The key challenge: reliability and robustness}

In preliminary design, the designer has to choose among all the different solutions. The best solution has to be optimal, non-dominated (according to Pareto optimality) and, last but not least, robust. Indeed, this last point is crucial in the framework of the preliminary design and is usually not considerate at this design stage.

The robustness of a product can be defined as the capacity of a product to maintain its performance values in spite of the changing in functioning conditions and the variability or the uncertainties of its parameters. G. Taguchi is regarded as the pioneer in robust design by the introduction of the signal to noise ratio.

A robust solution has to maximize the signal (performance value) and minimize the noise (scattering of the performance). The noise parameters come from the uncertainty or the variability of the input parameters or changing on functioning conditions.

In preliminary design context, these sources of uncertainties can be distinguished in two different classes:

- The aleatory or random uncertainty

- The epistemic uncertainty.

The aleatory uncertainty traduces the inherent variation of the physical system. Such variation corresponds to the random nature of the input parameters. They are usually modelled as random phenomenon. Its characterisation is generally difficult and needs a large amount of information. Most often such information does not exist and designers usually make assumptions for the characteristics of the mean, the variances and eventually the correlation coefficients. Then, probability distributions can be deduced from these data.

The second type, epistemic uncertainty, traduces the lack of knowledge or incomplete information. This uncertainty is reducible to aleatory uncertainty by understanding the design or by obtaining more relevant data. However, in practice it is too expensive to gather enough data to reduce the uncertainty. These uncertainty types are modelled using intervals with a lower and upper bound. This source of uncertainty is difficult to considerate with classical reliable tools due to the non random behaviour.

In the literature, two main approaches are proposed to take into consideration, during the optimization phase, these uncertainties:

- the Reliable Based Design Optimization (RBDO),

- and the Sequential Optimization and Reliability Assessment (SORA).

Both approaches are based on the calculation of the Most Probable Point of failure (MPPs). It consists in solving an optimization problem to find a reliability index on the MPPs and then to solve another optimization problem to find a best design.

The RBDO approach was developed to optimized design performance with low probability of failure for particular design problem. This method is composed of a double loop optimization problem. First, the optimization of the cost function is realized and then, the verification of the satisfaction of all the constraints for the desirable reliability is assessed.

The SORA approach is developed to improve the efficiency of the probabilistic optimization. It employs a single loop strategy with a series of cycles of deterministic optimization and reliability assessment.

These approaches are well adapted for classical design problem when the product is well defined. For preliminary design, even though it is possible to represent the stochastic uncertainty associated with few design variables and few parameters by probabilistic means, there are places where such a representation is not adequate. Indeed, some of the inputs are known:

- to exist in interval(s) and nothing else is known about the distribution, mean or variance,

- to correspond to a list of discrete values.

In this case, it is extremely difficult to estimate the reliability of the solution. Nowadays, it is clear that the existing tools and approaches must be adapted in the framework of the preliminary design. A possible strategy can be based on evolutionary optimization for the search of feasible and optimal solutions added by a sequential reliability analysis for instance by evaluation of the robustness of the design solution (e.g. by Monte Carlo analysis or interval analysis techniques).

The search for reliable is intrinsically much more complex and difficult than the classical search of feasible solution; the size of the search space is much bigger and must be explored globally. Designers are looking for reliable solution from technical and computational point of views (reliable computing). Robust design optimization problem may be regarded as constraint satisfaction problem with universally quantifiers and therefore are equivalent to Quantified Constraint Satisfaction Problem which are known as None Polynomial Hard problems.

This thematic issue of International Journal on Interactive Design and Manufacturing is concerned the "Decision support system in preliminary design". The different articles of this issue report different tools, methods and techniques involved in the subject that were selected by the editor-inchief with assistance of two guest editors. All individual articles cover one or more of the fields depicted in this editorial.

Finally the guest editors would thank all the contributors, the anonymous reviewers and the editor in chief for their dedicated work in publishing this thematic issue. 